\title{
BMJ Open Implementation of a community-based, physiotherapy-led, multidisciplinary model of care for the management of knee osteoarthritis: protocol for a feasibility study
}

\author{
Rebecca Livings (D) , ${ }^{1}$ Justine M Naylor, ${ }^{2,3}$ Kathryn Gibson, ${ }^{4}$ Sarah Dennis, ${ }^{3,5}$ \\ Jeanette Thom, ${ }^{6}$ Kathryn Mills, ${ }^{7}$ Siobhan M Schabrun (i) ${ }^{1}$
}

To cite: Livings R, Naylor JM, Gibson $\mathrm{K}$, et al. Implementation of a community-based, physiotherapy-led, multidisciplinary model of care for the management of knee osteoarthritis: protocol for a feasibility study. BMJ Open 2020;10:e039152. doi:10.1136/ bmjopen-2020-039152

- Prepublication history for this paper is available online. To view these files, please visit the journal online (http://dx.doi. org/10.1136/bmjopen-2020039152).

Received 07 April 2020

Revised 15 June 2020

Accepted 16 June 2020

Check for updates

(C) Author(s) (or their employer(s)) 2020. Re-use permitted under CC BY-NC. No commercial re-use. See rights and permissions. Published by BMJ.

For numbered affiliations see end of article.

Correspondence to

Rebecca Livings;

r.livings@neura.edu.au

\section{ABSTRACT}

Introduction There is a gap between the care people with knee osteoarthritis $(\mathrm{OA})$ should receive according to evidence-based guidelines and the care they do receive. This feasibility study aims to test the feasibility of developing and implementing a codesigned, physiotherapy-led, multidisciplinary, evidencebased model of care for knee $\mathrm{OA}$, among community physiotherapy practices in Australia, where community practice is defined as a professional physiotherapy business that is not controlled or paid for by the government.

Methods and analysis A mixed-methods quasiexperimental (pre/postintervention) study. In the preintervention phase, all consented physiotherapists working in nine metropolitan-based, community physiotherapy practices, and 26 patients with knee $0 \mathrm{~A}$ will be recruited. Patients will be recruited from all practices by the physiotherapists, using the outlined inclusion/exclusion criteria. An audit of physiotherapy treatment notes will occur using a proforma, to gain an understanding of current community physiotherapy treatment and documentation. Patient and physiotherapist interviews will be conducted to determine current practice for the management of knee OA. A codesign phase will follow, where a model of care will be developed by researchers, patients, clinical staff, members of the public and other stakeholders, based on current guidelines for conservative management of knee 0A. In the postintervention phase, a further 26 patients will be recruited, and the assessment process repeated to determine whether there is a change in practice. The feasibility outcome measures are: (1) number of patients who are recorded as receiving care according to current evidence-based guidelines; (2) number of patients who have patient-reported outcomes incorporated into their assessment and management plan; and (3) acceptability of the developed model to patients and physiotherapists. The clinical outcomes will include assessment of patient-reported outcome measures (pain, function, etc) in the preintervention and postintervention phases (baseline and 12 weeks) to assess trends towards change in participant symptoms.
Strengths and limitations of this study

- The study will rely on the highest level of evidence to benchmark current practice and inform the intervention.

- Physiotherapy practices that cover a diverse range of geographic regions, socioeconomic groups and physiotherapist experience levels will be included.

- The study will develop a codesigned conservative model of care involving researchers, patients, clinical staff, members of the public and other stakeholders, therefore, is more likely to be accepted by both providers and users, resulting in a higher rate of stakeholder satisfaction, continuous improvement and a reduced failure risk.

- The sample size is sufficient to provide the data required for potential future studies.

- Recall bias in patient and physiotherapist interviews is minimised.

Ethics and dissemination Ethical approval has been obtained from the University of New South Wales human ethics committee (approval number HC180864, approval period 6 February 2019 to 5 February 2024). The preintervention stage of this study is complete. The next stage is to implement the intervention and compare outcomes between the preintervention and postintervention phases. The results will be disseminated via peer-reviewed publications and presentations at conferences.

Trial registration number The preintervention phase of the study is retrospectively registered at ClinicalTrials. gov with registration number: ACTRN12620000188932. The intervention and postintervention phase of the study is prospectively registered at ClinicalTrials.gov with registration number: ACTRN12620000218998.

\section{INTRODUCTION}

Knee osteoarthritis (OA) is a degenerative joint disease affecting the cartilage and surrounding structures of the synovial joint. ${ }^{1}$ 
The condition results in pain, loss of muscle strength and disability, with detrimental effects on mental health and quality of life (QoL) $){ }^{1-3} \mathrm{OA}$ is the most common form of arthritis, ${ }^{4}$ with more than $15 \%$ of Australians affected. ${ }^{5}$ This figure is projected to rise to almost $25 \%$ by $2050 .^{5}$ As a result, OA is associated with enormous humanistic and economic burden, places substantial demands on healthcare systems ${ }^{46}$ and is recognised in Australia as a national health priority under the national chronic disease strategy. ${ }^{4}$

Arthritis is one of the most expensive disease groups in Australia, with health system expenditure reaching US $\$ 5.5$ billion in $2015 .^{7}$ By 2030 , the number of people with arthritis is projected to rise to 5.4 million and the associated health system cost increasing to AUS $\$ 7.6$ billion. $^{7}$ Hip and knee replacements for OA cost the health system approximately AUS $\$ 2.3$ billion in 2012/2013, and this figure is predicted to reach AUS $\$ 5.3$ billion by $2030 .^{7}$ In addition, arthritis affects a person's ability to work, ${ }^{8}$ making it the second most common cause of early retirement due to ill health, ${ }^{9}$ accounting for $40 \%$ of the loss in full-time employment and $42 \%$ loss in part-time employment due to chronic disease. ${ }^{10}$

The management of knee OA has been variable, and often limited to pharmaceutical management and total joint replacement. ${ }^{11}$ Recently, evidence-based recommendations for the management of knee OA have been developed by scientific societies and health organisations. ${ }^{312-15}$ Current best-practice guidelines for the treatment of knee OA focus on conservative (non-operative) multidisciplinary management that encompasses education, exercise, lifestyle modification and weight loss where relevant, while promoting self-management. ${ }^{312-14}$ This body of evidence demonstrates that multidisciplinary conservative management of knee $\mathrm{OA}$ is efficacious. However, conservative management can only be efficacious if it is implemented into everyday clinical practice. ${ }^{2}$

Numerous studies report a gap between the care people should receive based on best-practice guidelines and the care they do receive. ${ }^{16-18}$ In addition, there is considerable practice variation in the management of knee OA in Australia. ${ }^{19}$ For example, in the Bettering the Evaluation and Care of Health (BEACH) study, only $8.6 \%$ of people with $\mathrm{OA}$ were referred to physiotherapy by general practitioners, and this dropped to $4.6 \%$ when patients had a new presenting problem of arthritis. ${ }^{20}$ Further, in a systematic review of community-based observational studies comparing actual clinical practice with quality indicators of people with OA across the USA, UK, Norway, Canada, Australia, Portugal and Denmark, fewer than $40 \%$ of patients with OA were offered first-line non-pharmacological approaches,${ }^{21}$ which is considerably lower than the rate of referral for surgery $(\sim 78 \%) .{ }^{22}$ Given the strong evidence-base for the conservative management of knee OA, it is critical to address this practice gap.

Evidence-based physiotherapy programmes for the conservative management of knee OA have been trialled in both Australia and internationally. However, these programmes are either within the public health system for example, Osteoarthritis Chronic Care Programme (OACCP) ${ }^{3}$ supported by public healthcare system for example, Enabling Self-management and coping with Arthritic Pain using Exercise(ESCAPE-pain), ${ }^{23}$ or do not include the multidisciplinary team for example, Good Life with Osteoarthritis: Denmark (GLAD) ${ }^{24}$ The aim of this feasibility study is to test the feasibility of an evidencebased, physiotherapy-led, multidisciplinary model of care for knee OA which is developed and implemented using codesign, within the Australian community physiotherapy setting, which will also include the implementation of patient-reported outcome measures (PROMs). The clinical outcomes aim to examine the effect of the new model of care on patient-reported measures of knee stiffness, knee pain, knee function and polysymptomatic distress.

\section{METHODS}

\section{Study design}

This feasibility study will use a quasi-experimental, prepost design with an embedded qualitative component, to test the feasibility of implementing a codesigned model of care based on current best-practice guidelines for the management of knee $\mathrm{OA}$, in community physiotherapy practice. Specifically, the study will include: (1) a preintervention phase lasting approximately 6 months that will consist of an audit of physiotherapy treatment notes and patient and physiotherapist interviews to gain an understanding of current practice; (2) an intervention phase lasting approximately 2 months that will consist of codesigning a model of care in collaboration with researchers, patients, clinical staff, members of the public and other stakeholders, based on current guidelines for the conservative management of knee $\mathrm{OA}$; and (3) a postintervention phase lasting approximately 6 months that will replicate the preintervention stage and evaluate whether a change in practice has occurred (figure 1).

Within the study design outlined above, there are two phases in which clinical outcomes are assessed: the preintervention stage that establishes what community physiotherapy management for knee OA looks like now; and the postintervention stage that aims to establish whether community physiotherapists can feasibly adapt their care (from the initial starting point), so that it aligns with recommended practice, if it does not already.

Ethical approval has been obtained from the University of New South Wales human ethics committee (approval number HC180864).

\section{Study duration}

The study will be conducted over approximately 20 months, commencing March 2019.

\section{Study population and setting}

Nine physiotherapy practices in the Sydney area and 52 patients with knee OA (approximately $5-6$ per practice) will be recruited. These sample sizes are opportunistic, 


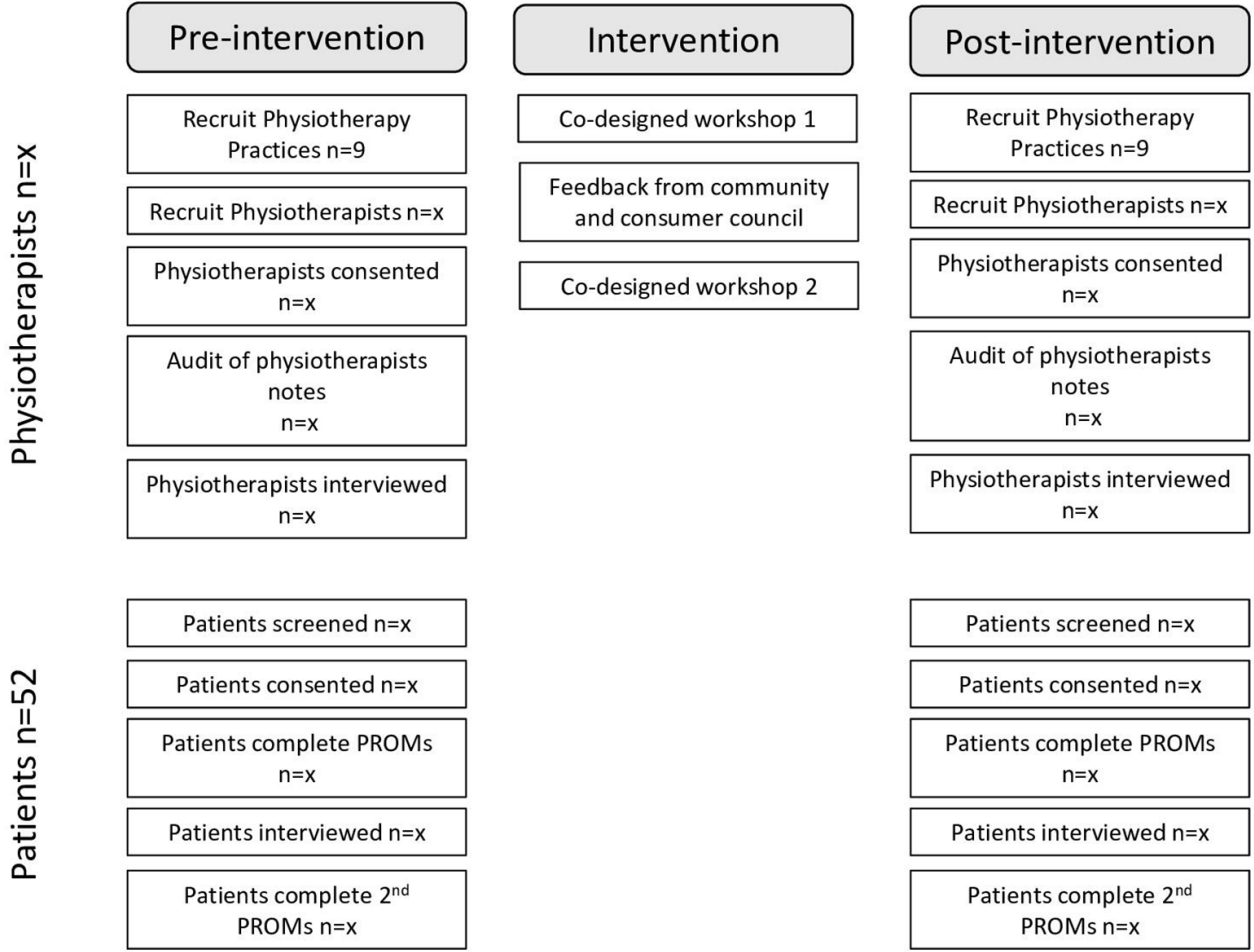

Figure 1 Flow diagram of the study. The recruitment of patients will occur after the practices and physiotherapists have been recruited and completed their baseline assessment and informed consent forms. Patients will only be considered to be enrolled in the trial after they have submitted their informed consent forms and baseline PROMs. PROMs, patient-reported outcome measures.

due to the study design being a small feasibility study, allowing the researchers to obtain data regarding the current study prior to a potential future larger study. While 12 people typically provide sufficient information for theme saturation in qualitative studies, ${ }^{25}$ we aim to enrol participants from each clinic in order to get a mix of socioeconomic backgrounds providing evidence of a trend in efficacy (if there is one), that will inform sample size calculations for future studies.

\section{Physiotherapy practices}

The practices will cover a wide range of geographic and socioeconomic profiles. Thus, observations should be generalisable across a number of settings.

\section{Physiotherapist participants}

All physiotherapists working in the practices who have given consent will be included in the study; they will be of any gender and age, with a range of experience levels.

\section{Patient participants}

Potential patients will be identified by the physiotherapists using the following inclusion and exclusion criteria.

Inclusion criteria: activity-related knee joint pain, morning stiffness $<30 \mathrm{~min}$ and aged over 45 years.

Exclusion criteria: inflammatory arthritis, gout, history or current infection in the joint, cognitive impairment, significant trauma, hemi or total knee replacement of the affected joint, knee surgery in the last 12 months, being treated under workers compensation, unable to understand English or unable to provide informed consent.

\section{Study procedures}

Figure 1 outlines the study phases. In brief, nine physiotherapy practices in the Sydney area and 52 patients with knee OA (approximately $5-6$ per practice) will be recruited. Physiotherapists and patients will be screened, and consent will be requested. The lead researcher (a physiotherapist) will interview physiotherapists using semistructured interviews either face-to-face or via telephone and will conduct an audit of physiotherapists treatment notes using a proforma, at the end of the preintervention phase and the end of the postintervention phase. Patients will complete PROMs at the time of recruitment and again 12 weeks after this, with up to three reminders in the case of non-return. The lead researcher (a physiotherapist) will interview patients using semistructured interviews either face-to-face or via telephone, approximately 6 weeks after recruitment in both the preintervention and postintervention phases.

The intervention will be codesigned in consultation with researchers, patients, clinical staff, members of the public and other stakeholders. Consultation for the codesign with the physiotherapists will occur during two workshops.

\section{Recruitment}

Practices and physiotherapists: practices already known to two investigators (ie, either working within them or related 
to workers within them) were invited to participate in the study. All consenting physiotherapists working in the practices will be recruited for the study. At least one site visit will be undertaken to gain an understanding of the layout of each practice. There will be at least one face-toface meeting with the physiotherapists to provide detailed description of the study, patient inclusion and exclusion criteria and gain written informed consent prior to study commencement.

Patients: patients will be recruited from all nine practices. The physiotherapists will identify patients using the outlined inclusion/exclusion criteria and gain verbal consent for the researcher to contact them by telephone. The researcher will contact each practice on a weekly basis to gain contact details for eligible patients. The researcher will contact all identified patients by telephone to confirm eligibility and discuss details of the study and patient participation. Eligible patients will be mailed a participant information statement and consent form and three PROMs-The Knee Osteoarthritis Outcomes Score (KOOS), the Multidimensional Health Assessment Questionnaire (MDHAQ) and the 2011 Fibromyalgia (FM) criteria questionnaire. Patients will return the completed questionnaires and signed consent form in a reply-paid envelope.

\section{Preintervention phase}

Consenting patients will be asked to complete the PROMs at initial recruitment and at 12 weeks (at a time when clinical improvement is expected to be clearly evident) ${ }^{26}$ The 12-week PROMs will be mailed out to participants, with up to three reminders in the case of non-return. After patients have consented, they will receive 'usual care', as per current practice at each clinic.

Six weeks after initial recruitment, the lead researcher will identify each patient's therapy records (physiotherapy treatment notes) and conduct a baseline audit of the physiotherapy knee OA management provided using a proforma. The lead researcher will conduct patient interviews following the audit of the therapy records and physiotherapist interviews will be conducted 6 weeks after the last patient is recruited.

The findings from the preintervention phase will inform the development of the codesigned model of care. They will also provide a snapshot of current practice in the community physiotherapy setting.

\section{Intervention phase-codesign of a guideline-based management approach to knee $\mathrm{OA}$}

The intervention will be codesigned in consultation with researchers, patients, clinical staff, members of the public and other stakeholders over a 2-month period. This will be done using the findings from the preintervention data, followed by consultation with all consented physiotherapists across two codesigned workshops. Further feedback will be gained from community representatives from the Sydney Partnership for Health, Education, Research and
Enterprise (SPHERE) and the Community and Consumer Council (CCC) for Musculoskeletal Health.

\section{Postintervention phase}

A further 26 patients will be recruited, over a period of 3-6 months, from the same practices as the preintervention phase. The same approach to recruitment, patient assessment, audit of notes and interviews will be taken as described in the preintervention period.

\section{Audit of treatment notes}

An audit of physiotherapy treatment notes will be conducted, to extract data using a proforma developed based on recommended guidelines for the conservative management of knee OA including those from the European League Against Rheumatism recommendations for the non-pharmacological core management of hip and knee osteoarthritis, ${ }^{12}$ OA Research Society International guidelines for the non-surgical management of knee osteoarthritis, ${ }^{13}$ National Institute for Health and Care Excellence, Osteoarthritis: care and management in adults, Clinical Guideline ${ }^{14}$ and Royal Australian College of General Practitioners: Guideline for the management of knee and hip osteoarthritis, Second edition. ${ }^{15}$ Data collected through the audit of treatment notes in the preintervention stage will be recorded in Microsoft Excel (Office 365) spreadsheets and analysed using descriptive statistics (mean, SD and proportion) as follows: the proportion of patients who received each of the core guideline treatments and the proportion of patients who were referred to another healthcare professional for each of the core guideline treatments.

An audit of postintervention treatment notes will test for change in treatment due to the intervention and will be compared against care that is recommended in guidelines for the conservative management of knee OA. Descriptive statistics of the patient will determine whether all core guideline treatments need to be addressed. For example, if the patient is obese, weight management should be discussed, and a referral to a dietician or at least discussion regarding dietetic input must be made. However, if the patient has a body mass index $<25$, this treatment recommendation would be nullified.

\section{Semistructured interviews}

Patients will be interviewed in a one-to-one format, either face-to-face or by telephone as possible. Their experience and perspectives of how their knee OA was managed will be gathered using a semistructured face-to-face interview at 6 weeks postrecruitment and will be recorded and transcribed verbatim.

Physiotherapist interviews will be in a one-to-one format, either face-to-face or by telephone as possible. The semistructured interviews of physiotherapists will provide the following information: the physiotherapists' perceptions of their current management of knee OA; perceived barriers to providing the best physiotherapy management of knee OA; how patients are assessed; how 
Table 1 Feasibility outcome measures

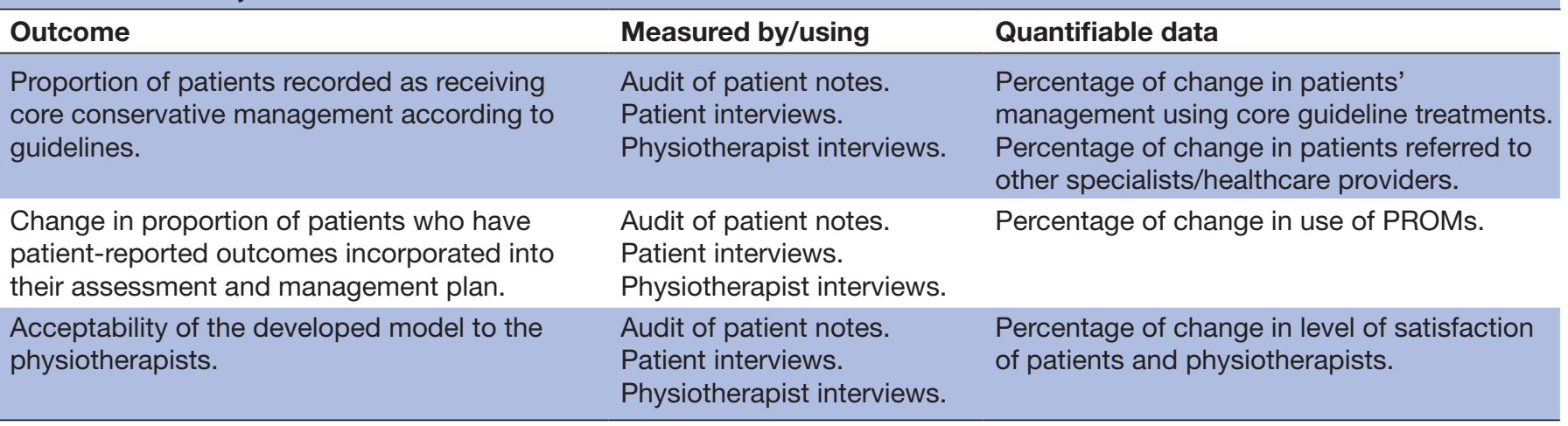

PROMs, patient-reported outcome measures.

satisfied physiotherapists are with their current management of knee OA; and how they perceive this could be improved. The interviews will be digitally recorded and transcribed verbatim.

Thematic analysis of both patient and physiotherapist interviews will be undertaken. The transcripts will be transcribed and coded in NVivo. ${ }^{27}$ The coding will be discussed with the research team and revised if necessary. The codes will then be organised into broad themes that reflect experiences and perceptions of the patients and physiotherapists.

\section{Codesign workshops}

Two workshops will be held in order to inform the codesign of the intervention and all recruited physiotherapists will participate in both the workshops. The first workshop will highlight what the current guidelines are for the conservative management of knee OA, and aspects of practice that deviate from or align with these recommended guidelines, based on deidentified patient commentary on how individuals with knee OA perceived the treatment they received, and data extracted in the audit of physiotherapy treatment notes. Discussion regarding the benefits and limitations of PROMs and their availability for use will be included. The current use of exercise therapy and multidisciplinary input will be reviewed, and consensus sought on appropriate practice changes to be implemented if necessary, to align with current guidelines.

The second workshop will consist of presentation of a draft model of the intervention, based on data gathered

\section{Table 2 Clinical outcomes}

\begin{tabular}{ll}
\hline Outcome & Measurement tool \\
\hline Knee stiffness & KOOS \\
Knee pain & KOOS, MDHAQ \\
Knee function & KOOS, MDHAQ \\
$\begin{array}{l}\text { Polysymptomatic } \\
\text { distress }\end{array}$ & $\begin{array}{l}\text { S011 FM criteria questionnaire (2011 FM } \\
\text { Survey) }\end{array}$ \\
\hline
\end{tabular}

FM, Fibromyalgia; KOOS, Knee Osteoarthritis Outcomes Score; MDHAQ, Multidimensional Health Assessment Questionnaire. from the first workshop. Further feedback will be gathered. There will be discussion regarding evidence and strategies for implementation of the intervention and role-play will be used to develop the physiotherapists' confidence to deliver the intervention and improve knowledge as described in studies such as Shen $e t a l^{28}$

The outcome of the two codesign workshops will be consensus in relation to the final intervention and its implementation.

\section{Patient-reported outcome measures}

PROMs are defined as 'any report of the status of a patient's health condition that comes directly from the patient, without interpretation of the patient's response by a clinician or anyone else. ${ }^{29}$ Physiotherapists are increasingly encouraged to use PROMs to demonstrate response to treatment and encourage patient-centred care. ${ }^{30}$ For this study, three measures have been selected: 1. The KOOS, ${ }^{31}$ which was developed and validated to specifically assess the course of knee injury and treatment outcome and is an appropriate condition-specific measure. The KOOS includes 42 items within five subscales Pain (nine items); Symptoms (seven items); Activities of Daily Living (ADL), Function (17 items); Sport and Recreation Function (five items); Quality of Life (four items). The KOOS will provide an indication of change in knee-specific pain and function.

2. The MDHAQ ${ }^{32}$ which was designed to improve the quality of clinical care and assess a range of patient outcomes including physical function, pain and patient global assessment of disease activity. It provides a more holistic evaluation of the impact of disease on the patient than a disease-specific measure. The MDH$\mathrm{AQ}$ includes 10 queries concerning activities of daily living to evaluate physical function (FN), pain, patient global assessment (PATGL) and fatigue. RAPID3 is a composite index that includes $\mathrm{FN}$, pain and PATGL. The MDHAQ will provide an indication of change in pain and function more generally in the preintervention and postintervention phases of the study.

3. The 2011 FM criteria questionnaire ${ }^{33}$ was developed for epidemiologic and clinical studies to allow patients 
to be classified into FM and non-FM groups. It also provides a continuous measure of the degree of 'fibromyalgianess' the patient experiences. The 2011 FM criteria consist of two scales: the Widespread Pain Index and the Symptom Severity scale. The FM criteria will provide an indication of the proportion of patients classified as meeting FM criteria and will inform interpretation of the other two PROM measures.

Preliminary assessment of the feasibility of implementation of PROMs questionnaires in this setting will be conducted by assessing the increased uptake of PROMs by the physiotherapists: this will be ascertained through the audit of physiotherapy treatment notes.

\section{Patient and public involvement}

Patients, clinical staff, members of the public and other stakeholders will be involved at several stages of the trial, including the design, and development and delivery of the implementation for the study. In the planning stage various stakeholders were engaged to help establish the design of the study. We will receive feedback from patients about their thoughts of how their knee OA could be managed, and from physiotherapists about how they could improve practice. The intervention will be codesigned in consultation with researchers, patients, clinical staff, members of the public and other stakeholders including representatives from the (SPHERE) and the CCC for Musculoskeletal Health. Patients will be invited to request study results if interested.

\section{OUTCOMES AND ANALYSIS}

\section{Feasibility outcome measures}

The three feasibility outcome measures, the means by which they are measured and the data that will be produced are provided in table 1 , where the satisfaction of patients will be assessed using a unipolar scale of 1-10, and physiotherapists' confidence in how they deliver treatment will be assessed using a point Likert scale.

\section{Clinical outcomes}

Clinical outcomes will be assessed through a comparison of the change in PROMs between initial recruitment and at 12 weeks (table 2). Additionally, demographic and clinical characteristics will be collected. The mean change (95\% CI) in PROM scores across time from the pooled data of the two cohorts will also be presented. As this study is not set up as a randomised controlled trial, there will be no comparison between the PROMs collected from the preintervention and postintervention cohorts.

\section{DISCUSSION}

The prevalence of knee OA is growing with the ageing population. Since there is no cure for knee OA, a conservative management model of care to alleviate symptoms is required in community practice, enabling people to receive evidence-based care, live better with knee $\mathrm{OA}$ and reduce demand for knee replacement surgery. Although multidisciplinary conservative treatment of knee OA can improve the management of symptoms ${ }^{34}$ and evidencebased recommendations for the management of OA have been developed by scientific societies and health organisations, ${ }^{3}{ }^{313}$ evidence suggests that these have not been implemented in the community physiotherapy setting. ${ }^{20}$ This feasibility study will establish whether a communitybased, physiotherapy-led multidisciplinary model of care for managing knee $\mathrm{OA}$ can be developed and imple$\underset{35}{\operatorname{mented}}$ in the community physiotherapy setting.

In conclusion, this study will yield important data about current practice for the management of knee OA in the community physiotherapy setting and the feasibility of implementing a codesigned evidence-based model of care for the management of knee OA in this setting. Data obtained are expected to inform the development of a future large-scale research trial.

\section{Study status}

The preintervention phase of this study began patient recruitment in March 2019. The postintervention phase of this study is expected to start recruitment in March 2020 .

\section{ARTICLE SUMMARY}

Arthritis is one of the most expensive disease groups in Australia. The management of knee OA has been variable, often results in total joint replacement, which is predicted to cost the health system AUS $\$ 5.3$ billion by 2030. Evidence-based recommendations for the multidisciplinary conservative management of knee OA have been shown to be efficacious.

This feasibility study aims to observe current community physiotherapy practice in the management of knee $\mathrm{OA}$ and test the feasibility of developing and implementing a codesigned, physiotherapy-led, multidisciplinary, evidence-based model of care in this setting.

\section{Strengths and limitations}

The strengths of this study include: (1) reliance on the highest level of evidence to benchmark current practice and inform the intervention; (2) inclusion of physiotherapy practices that cover a diverse range of geographic regions, socioeconomic groups and physiotherapist experience levels; and (3) the development of a codesigned conservative model of care involving patients, clinical staff, members of the public and other stakeholders is more likely to be accepted by both providers and users, resulting in a higher rate of stakeholder satisfaction, continuous improvement and a reduced failure risk.

Although the study's size is small, this is a limitation, rather than a weakness, as it is the right size for the purpose of the study and will provide the information needed for potential future studies. Recall bias could occur, although this study is not a high risk due to the subject manor and 
study design with objective medical record data being sought, and the interviews exploring what people think, therefore it is not a weakness within this study.

\section{Ethics and dissemination}

The Study received full Ethical approval from the University of New South Wales human ethics committee (approval number HC180864) on 06 February 2019, prior to commencing recruitment which is ongoing.

The trial will be reported in accordance with the CONSORT guidelines. ${ }^{36} 37$ The results will be submitted to high impact peer-reviewed journals with authorship following International Committee of Medical Journal Editors recommendations and will be presented at national and potentially international conferences. The investigators will share Individual deidentified participant data on reasonable request to the chief investigator.

\section{Author affiliations}

${ }^{1}$ Neuroscience Research Australia, Randwick, New South Wales, Australia

${ }^{2}$ South West Sydney Clinical School, University of New South Wales, Sydney, New

South Wales, Australia

${ }^{3}$ Ingham Institute for Applied Medical Research, Liverpool, New South Wales, Australia

${ }^{4}$ Rheumatology Department, Liverpool Hospital, Liverpool, New South Wales, Australia

${ }^{5}$ Clinical and Rehabilitation Sciences, Faculty of Health Sciences, University of Sydney, Sydney, New South Wales, Australia

${ }^{6}$ School of Medical Sciences, University of New South Wales, Kensington, New South Wales, Australia

${ }^{7}$ Department of Health Professions, Macquarie University, Sydney, New South Wales, Australia

\section{Twitter Siobhan M Schabrun @DrSMSchabrun}

Acknowledgements This study was supported by SPHERE STREAM Health CAG.

Contributors RL is the principal investigator. RL, SMS, KG and JMN conceived the project idea and design. JT, SD and KM provided specialist expertise and will continue to do so throughout the study. All authors have read and given final approval of the protocol.

Funding This study is funded by SPHERE STREAM Health CAG. SS receives salary support from The National Health and Medical Research Council of Australia (grant number 1105040).

Disclaimer The lead author (the manuscript's guarantor) affirms that the manuscript is an honest, accurate and transparent account of the study being reported; that no important aspects of the study have been omitted; and that any discrepancies from the study as originally planned (and, if relevant, registered) have been explained.

Competing interests All authors have completed the ICMJE uniform disclosure form at www.icmje.org/coi_disclosure.pdf and declare no support from any organisation for the submitted work; no financial relationships with any organisations that might have an interest in the submitted work in the previous three years; no other relationships or activities that could appear to have influenced the submitted work.

Patient and public involvement Patients and/or the public were involved in the design, or conduct, or reporting or dissemination plans of this research. Refer to the Methods section for further details.

Patient consent for publication Not required.

Provenance and peer review Not commissioned; externally peer reviewed.

Open access This is an open access article distributed in accordance with the Creative Commons Attribution Non Commercial (CC BY-NC 4.0) license, which permits others to distribute, remix, adapt, build upon this work noncommercially, and license their derivative works on different terms, provided the original work is properly cited, appropriate credit is given, any changes made indicated, and the use is non-commercial. See: http://creativecommons.org/ licenses/by-nc/4.0/.

Author note The corresponding author has the right to grant on behalf of all authors and does grant on behalf of all authors, an exclusive licence (or nonexclusive for government employees) on a worldwide basis to the BMJ Publishing Group Ltd to permit this article (if accepted) to be published in BMJ editions and any other BMJPGL products and sublicences such use and exploit all subsidiary rights, as set out in our licence.

\section{ORCID iDs}

Rebecca Livings http://orcid.org/0000-0001-5079-7351

Siobhan M Schabrun http://orcid.org/0000-0002-9083-3107

\section{REFERENCES}

1 Cakar M, Ayanoglu S, Cabuk H, et al. Association between vitamin $\mathrm{D}$ concentrations and knee pain in patients with osteoarthritis. PeerJ 2018;6:e4670.

2 Bennell KL, Hunter DJ, Hinman RS. Management of osteoarthritis of the knee. BMJ 2012;345:e4934. and.

$3 \mathrm{ACl}$ Musculoskeletal Network. Osteoarthritis chronic care program model of care. Agency for clinical innovation: Sydney, 2012. Available: https://www.aci.health.nsw.gov.au/_data/assets/pdf_file/ 0003/165306/Osteoarthritis-Chronic-Care-Program-Mode-of-CareHigh-Resolution.pdf

4 Schofield D, Shrestha R, Cunich M. Counting the cost Part 2 economic costs. The current and future burden of arthritis. The University of Sydney report prepared for arthritis Australia; 2016.

5 Australian Institute of Health and Welfare. A picture of osteoarthritis in Australia. Arthritis series no. 5. Cat. no. PHE 93. Canberra: AlHW, 2007.

6 Hay MC, Weisner TS, Subramanian S, et al. Harnessing experience: exploring the gap between evidence-based medicine and clinical practice. J Eval Clin Pract 2008;14:707-13.

7 Ackerman IN, Bohensky MA, Zomer E, et al. The projected burden of primary total knee and hip replacement for osteoarthritis in Australia to the year 2030. BMC Musculoskelet Disord 2019;20:90.

8 National Strategic Action Plan for Arthritis Commonwealth of Australia as represented by the Department of Health. The National strategic action plan for arthritis ISBN: 978-0-64833-27-9-4; 2019.

9 Schofield DJ, Shrestha RN, Cunich M, et al. Lost productive life years caused by chronic conditions in Australians aged 45-64 years, 20102030. Med J Aust 2015;203:260.

10 Australian Institute of Health and Welfare. Chronic disease and participation in work.Cat. No. PHE109 2009.

11 Hunter DJ. Lower extremity osteoarthritis management needs a paradigm shift. Br J Sports Med 2011;45:283-8.

12 Fernandes L, Hagen KB, Bijlsma JWJ, et al. EULAR recommendations for the non-pharmacological core management of hip and knee osteoarthritis. Ann Rheum Dis 2013;72:1125-35.

13 Bannuru RR, Osani MC, Vaysbrot EE, et al. OARSI guidelines for the non-surgical management of knee, hip, and polyarticular osteoarthritis. Osteoarthritis Cartilage 2019;27:1578-89.

14 NICE. Osteoarthritis: care and management in adults. clinical guideline CG177. National clinical guideline centre. National Institute for health and clinical excellence, 2014a. Available: http://www.nice. org.uk

15 The Royal Australian College of General Practitioners. Guideline for the management of knee and hip osteoarthritis. 2nd edn. East Melbourne, Vic: RACGP, 2018.

16 Balas E, Boren S, Managing Clinical Knowledge for Health Care Improvement. Yearbook of medical informatics. Stuttgart: Schattauer Verlagsgesellschaft mbH, 2000: 65-70.

17 Grant J, Cottrell R, Cluzeau F, et al. Evaluating "payback" on biomedical research from papers cited in clinical guidelines: applied bibliometric study. BMJ 2000;320:1107-11.

18 Wratschko K. Empirical Setting: The pharmaceutical industry. In: Strategic orientation and alliance Portfolio configuration. New York, NY: Springer, 2009.

19 Clinical care standards - Osteoarthritis of the Knee - the Case for Improvement, 2018. Available: https://www.safetyandquality.gov.au/ wp-content/uploads/2018/04/Osteoarthritis-of-the-Knee-the-Casefor-Improvement.pdf

20 Dennis S, Watts I, Pan Y, et al. The likelihood of general practitioners referring patients to physiotherapists is low for some health problems: secondary analysis of the Bettering the evaluation and care of health (BEACH) observational study. J Physiother 2018;64:178-82. 
21 Hagen KB, Smedslund G, Østerås N, et al. Quality of communitybased osteoarthritis care: a systematic review and meta-analysis. Arthritis Care Res 2016;68:1443-52.

22 Basedow M, Esterman A. Assessing appropriateness of osteoarthritis care using quality indicators: a systematic review. J Eval Clin Pract 2015;21:782-9.

23 Sibley F, Thompson F, Carter A, et al. Tackling inactivity and osteoarthritis through a health and community leisure partnership. Perspect Public Health 2018;138:188-9.

24 Skou ST, Roos EM. Good Life with osteoArthritis in Denmark (GLA:D ${ }^{T M}$ ): evidence-based education and supervised neuromuscular exercise delivered by certified physiotherapists nationwide. BMC Musculoskelet Disord 2017;18:72.

25 Guest G, Bunce A, Johnson L. How many interviews are enough? An experiment with data saturation and variability. Field Methods 2006;18:59-82.

26 Busch AJ, Barber KAR, Overend TJ, et al. Exercise for treating fibromyalgia syndrome. Cochrane Database Syst Rev 2007;4:CD003786.

27 NVivo qualitative data analysis software; QSR international Pty LTD. version 122018

28 Shen Z, Jiang C, Chen L. Evaluation of a train-the-trainer program for stable coronary artery disease management in community settings: a pilot study. Patient Educ Couns 2018;101:256-65.

29 U.S. Department of Health and Human Services FDA Center for Drug Evaluation and Research, U.S. Department of Health and Human Services FDA Center for Biologics Evaluation and Research, U.S. Department of Health and Human Services FDA Center for Devices and Radiological Health, et al. Guidance for industry: patient- reported outcome measures: use in medical product development to support labeling claims: draft guidance. Health Qual Life Outcomes 2006;4:79.

30 Kyte DG, Calvert M, van der Wees PJ, et al. An introduction to patient-reported outcome measures (PROMs) in physiotherapy. Physiotherapy 2015;101:119-25.

31 Roos EM, Stefan Lohmander L. The knee injury and osteoarthritis outcome score (KOOS): from joint injury to osteoarthritis. Health Qual Life Outcomes 2003;64.

32 Pincus T, Maclean R, Yazici Y, et al. Quantitative measurement of patient status in the regular care of patients with rheumatic diseases over 25 years as a continuous quality improvement activity, rather than traditional research. Clin Exp Rheumatol 2007;25:69-81.

33 Wolfe F, Clauw DJ, Fitzcharles M-A, et al. Fibromyalgia criteria and severity scales for clinical and epidemiological studies: a modification of the ACR preliminary diagnostic criteria for fibromyalgia. J Rheumatol 2011;38:1113-22.

34 Health economics and evaluation team OACCP: monitoring and evaluation plan 2017.

35 Steen M, Manschot M, De Koning N. Benefits of Co-design in service design projects. IJDesign 2011;5.

36 Eldridge SM, Chan CL, Campbell MJ, et al. Consort 2010 statement: extension to randomised pilot and feasibility trials. BMJ 2016;355:i5239.

37 Thabane L, Hopewell S, Lancaster GA, et al. Methods and processes for development of a consort extension for reporting pilot randomized controlled trials. Pilot Feasibility Stud 2016;2:eCollection 2016. 